\title{
ARQUITECTURA, ARTE FUNCIONAL
}

\section{(ARCHITECTURE, A FUNCTIONAL ART)}

\author{
Juan Monjo Carrió, Dr. Arquitecto \\ Catedrático de Construcción \\ Escuela T. S. de Arquitectura \\ Universidad de Valladolid
}

\section{RESUMEN}

Se inicia el trabajo analizando los conceptos de Arte, Ciencia y Técnica y su evolución histórica, distinguiendo entre las "bellas artes" y las "artes técnicas". A continuación se definen los conceptos de Arquitecto y Arquitectura, tanto conceptual como profesionalmente, analizando, asimismo, su evolución histórica y haciendo hincapié en la interdependencia entre la concepción arquitectónica como "bella arte" y la tecnología constructiva como "arte técnica", para terminar recordando la necesaria base científica de esta última (la Física de la Construcción). Como consecuencia, se recuerda la necesidad de los conocimientos de la tecnología constructiva en los arquitectos profesionales. Por último, se analiza el carácter funcional de la Arquitectura (Arquitectura como "arte funcional") y se hace un breve recorrido por los tres aspectos básicos de esa funcionalidad (Integridad-firmitas, Habitabilidad-utilitas y Estética-venustas).

\section{SUMMARY}

The begining of this work is devoted to the analysis of the concepts of Art, Science and Technique and their historical evolution, distinguishing between "fine arts" and "technique arts". Following, Architect and Architecture terms are defined both conceptual and professionally, analysing as well its historical evolution and pointing out the interdependence between the architectural conception as "fine art" and the constructive technology as "technique art", finally reminding the necessary scientific base of this one (Construction Physics). Consequently, the need for architecture professionals of constructive technology knowledge, is also reminded. At last, the functional character of the Architecture (Architecture as a "functional art") is analysed, going over the three basic aspects of this functionality (Integrity-firmitas, Habitability-utilitas and Aesthetics-venustas).

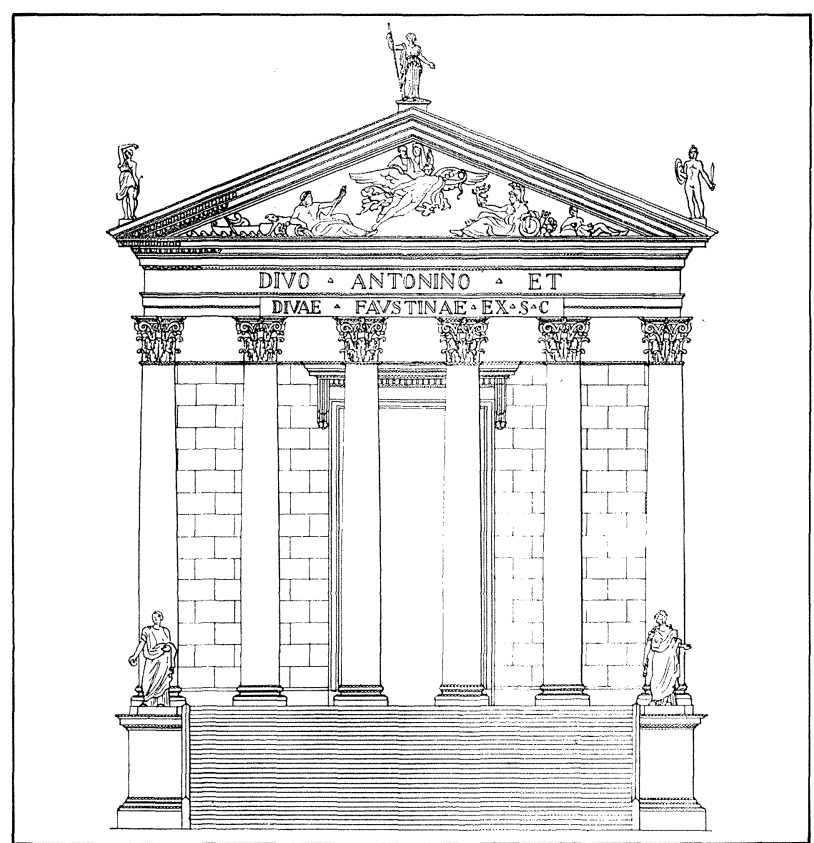

INTRODUCCION. BELLAS ARTES Y ARTES TECNICAS

Histórica y filosóficamente las artes se han reunido en dos grandes grupos, cada uno de ellos con su calificativo específico que ilustra sobre su base, su instrumentación y sus objetivos. Así tenemos, por un lado, el grupo de las «Bellas Artes» (Artes Bellas), cuyo objetivo y preocupación fundamental es la belleza aparente, por lo que se deben incluir en el reino de la estética. Por otro lado distinguimos las «Artes Técnicas», que, sin olvidar la belleza, se orientan y pertenecen al mundo de la Tecnología, utilizando toda la creatividad potencial que la ciencia de las técnicas («tecno»-«logos») Ileva consigo.

La Historia y la Sociedad han distinguido claramente entre estos dos tipos de arte asimilando las primeras a las ciencias humanísticas y las segundas a las ciencias técnicas que, a la larga, han acaparado el uso de la palabra Ciencia, quedando para las primeras el de su calificativo, Humanidades.

Pero no debemos perder de vista que en su origen todas eran ciencias, pues se basaban en el estudio de los principios y las causas, por un lado, de las potencias anímicas $y$, por otro, de los hechos naturales, y estas ciencias desarrollaban de algún modo la creatividad implícita en dichas potencias y hechos $y$, por lo tanto, la capacidad artística que el hombre podía obtener de ellas.

\section{Ciencias y Artes}

Es más, la Historia nos enseña que el pensamiento primitivo tendía a distinguir en dos grupos las actividades del hombre, estableciendo dos niveles: El 


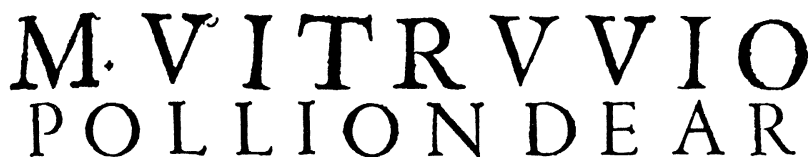

C H I T E C T V R A, DIVIDIDO EN diez libros, traduzidos de Latin en Caftellano por Miguel de Vrrea Architecto,y facadoen fu perfectió por Iuan Gracian impreffor vezino de Alcala.

DIRIGIDO ALA S. C. R. M. DEL REY DON PHElippe Segundo defte nombre nuestro Señor.

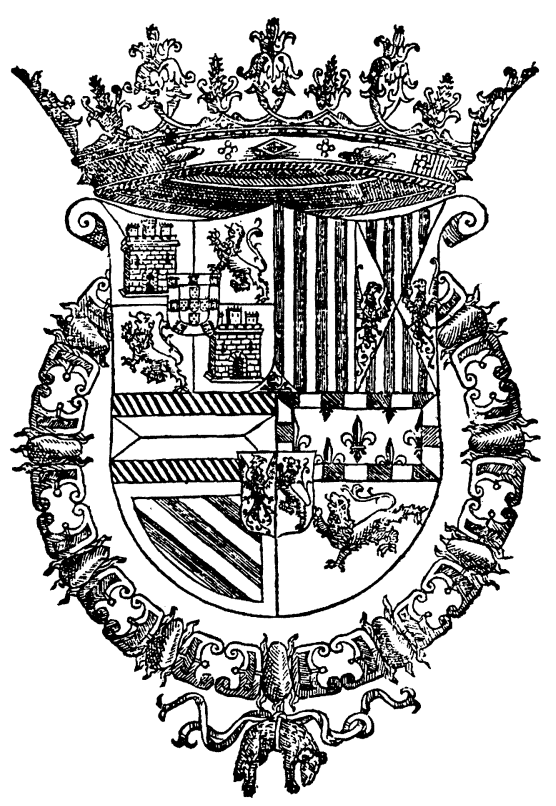

CON PRIVILEGIO.

Impreffo en Alcala de Henares por Iuan Gracian. Año. M. D. LXXXII.

De Architectura

Marco Vitrubio Polión.

Versión de Miguel de Urrea. Madrid, 1582

Edición Facsimil, Albatros Ediciones, 1978

superior, en el que se hallaban la Religión y la Ciencia (religiosos y filósofos) que basaban su actividad en el uso de las potencias anímicas. El inferior, en el que se localizaban las técnicas y las artes (técnicos y artífices) cuya actividad estaba basada fundamentalmente en las capacidades y habilidades manuales.

Poco a poco estos dos niveles se fueron fundiendo, o cuanto menos solapando, a medida que las ciencias y las técnicas se apoyaban mutuamente para seguir desarrollándose, no perdiendo por ello su identidad.

\section{Artes y Técnicas}

También ocurrió una mutación interesante que nos afecta más directamente. Esas artes, que en un principio eran más bien oficios y se fundían con las técnicas, a medida que el hombre profundizó en su estudio (les aplicó los principios científicos) se fue- ron desdoblando, cada una, en dos ramas que se complementaban: la de la creatividad pura y la de la creatividad aplicada, desarrollándose más una u otra rama, según el objetivo final de cada arte. Es entonces cuando podemos considerar que aparece más claramente la distinción entre Arte Bella y Arte Técnica que, sin dejar de estar íntimamente relacionadas, sufren un desarrollo individual que las significa.

En la Arquitectura este proceso evolutivo ha sido muy patente e incluso se ha llegado a reflejar en disposiciones legales. Tal es el caso de la reorganización de los estudios artísticos en España, del año 1844, en el que los de Arquitectura se dividieron en dos ramas, la de la Bella Arquitectura (Bella Arte) y la del Arte o Ciencia de Construir (Arte Técnica), cada una apoyada necesariamente en la otra.

Esta división histórica entre Bella Arte y Arte Técni$\mathrm{ca}$, se reflejó, asimismo, en la denominación de los sujetos de ambas, de tal modo que se utilizó el calificativo de "artista" para el de las bellas artes y el de "artífice» (de "ars fácere», el que hace el arte) para el de las artes técnicas.

En muchos casos, en la mayoría, ambos calificativos son aplicables al mismo sujeto. El pintor o el escultor crean la idea de sus obras y, además, las realizan. La creación sigue prácticamente un solo proceso, del principio al fin; el compositor de música, sin embargo, crea su partitura, pero la interpretación y ejecución corren a cargo de otro sujeto, el director de orquesta o el intérprete solista, que puede, o no, ser la misma persona. Algo similar ocurre en la Arquitectura, donde un profesional crea el proyecto y otro lo lleva a cabo (lo dirige).

Sin embargo, la realidad nos dice que la sociedad y la legislación actuales en nuestro país exigen del arquitecto que sea capaz de hacer las dos funciones, es decir, que pueda ser artista y artífice a la vez y, por consiguiente, que esté inmerso tanto en el mundo de la Arquitectura como bella arte, como en el de la Tecnología Constructiva como arte técnica.

\section{Arte funcional}

Por otra parte, todas las artes tienen una característica específica que las individualiza indicando su campo de acción y, sobre todo, sus objetivos finales, y esta característica es la que origina, precisamente, la relación de interdependencia entre los aspectos estéticos y tecnológicos de cada arte, ya que establece la tecnología necesaria para la consecución apropiada del producto artístico final en cada caso.

Resulta pues fundamental, delimitar, de la manera más precisa, cuál es la mencionada característica para poder establecer las bases de la creatividad tecnológica de cada una de las artes. 
En nuestro caso, así como la Pintura es fundamentalmente visual o la Música es básicamente auditiva, podemos apreciar que la Arquitectura es una bella arte «funcional».

La Arquitectura - cada arquitectura concreta - se crea, no sólo para ser vista, sino, sobre todo, para ser vivida, para ser utilizada.

El calificativo de funcional tiene pues aquí un sentido de uso, de utilización vital, pues no sólo se usa la arquitectura como se puede usar un objeto más o menos manual, sino cada arquitectura tiene que vivirse desde dentro, de tal manera que no tiene sentido una "arquitectura» sólo para ser vista y mucho menos sobre papel; entraríamos en los campos de la Pintura o de la Escultura.

La Arquitectura, en definitiva, tiene que diseñarse para ser vivida y, por ello, tiene que proyectarse para poder ser construida. $Y$ aquí nos encontramos con la faceta tecnológica del arte (la ciencia) de construir. Ese objetivo de esta bella arte, que es a la vez su calificativo - la funcionalidad- necesita para su logro de un arte técnico que sea capaz de hacer construible ( $y$, por tanto, utilizable) lo que la bella arte ha creado. Necesita, pues, de la creatividad de la tecnología para que la creatividad funcional que anima el espíritu de la Arquitectura tenga su realización concreta.

Analicemos concretamente esta división de las artes en el caso de la Arquitectura. Para ello, veamos los conceptos de Arquitecto y de Tecnología Constructiva.

\section{EL ARQUITECTO COMO ARTISTA Y COMO ARTIFICE}

El diccionario de la Real Academia Española de la Lengua define al arquitecto como «el que profesa o ejerce la Arquitectura». Y más adelante define la Arquitectura como el «arte de proyectar y construir edificios».

Por otra parte, muchas son las definiciones que se pueden dar del arquitecto, según la época históri$c a$, según la zona cultural y según la formación del que la da, pero hay una, que es quizá la más universal, que en mi opinión es la más acertada y que, al fin y al cabo, se puede deducir filológica y etimológicamente de la propia palabra. Arquitecto viene de las palabras griegas "archo" y «tecton» (mandar y técnicos), es decir, el que está por encima de ellos, el que los coordina y dirige. Podríamos, pues, en este sentido, definir al arquitecto como "coordinador y director del proceso constructivo de un edificio y, por tanto, de todos los técnicos especialistas que en él intervienen, desde los diseñadores o proyectistas iniciales hasta los decoradores finales, pasando por los fabricantes de materiales y compo-

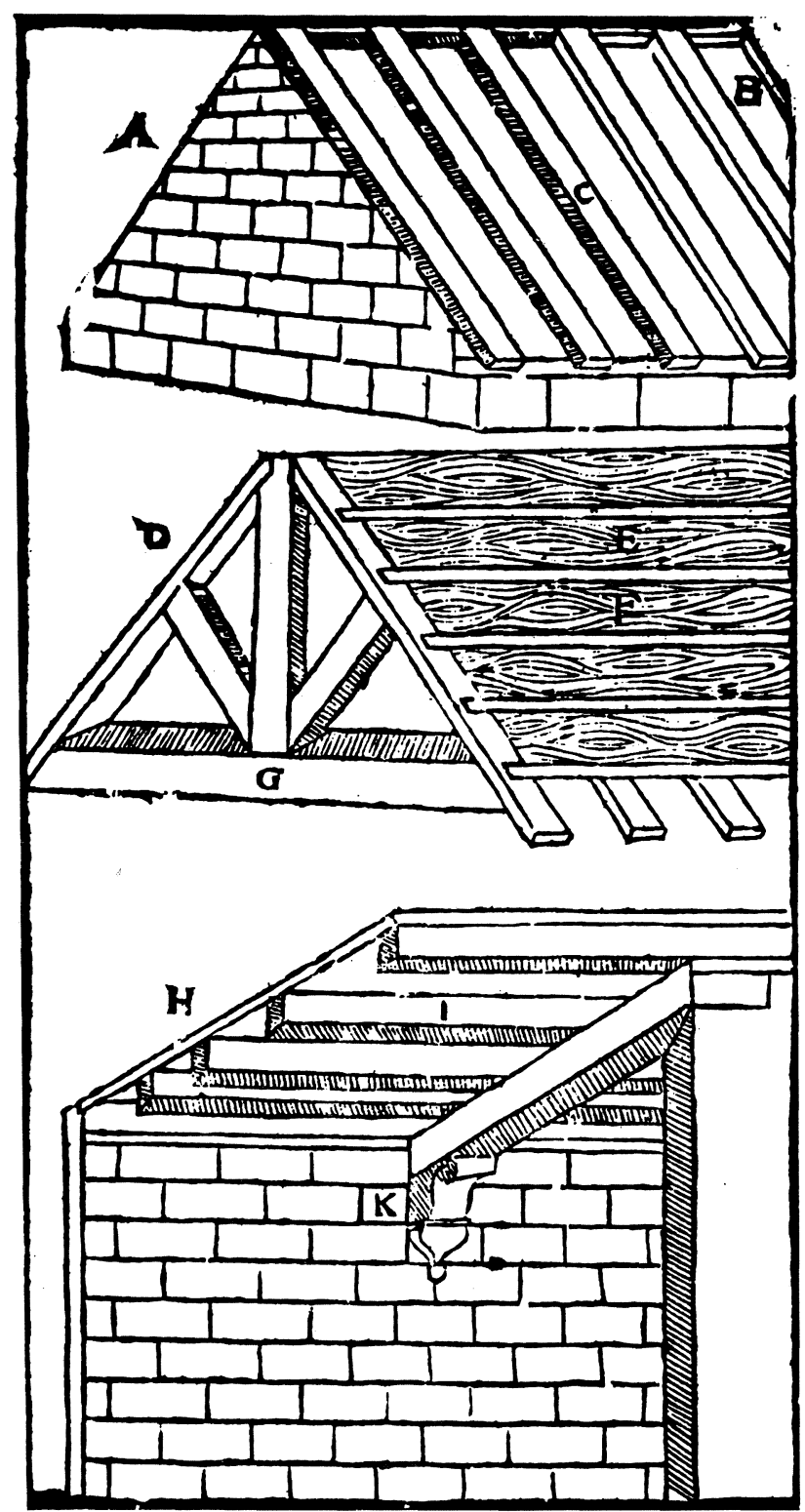

De Architectura

Marco Vitrubio Polión. Libro quarto

Versión de Miguel de Urrea. Madrid, 1582

Edición Facsimil, Albatros Ediciones, 1978

nentes y los constructores o ejecutores materiales del edificio». Es, pues, el "super-técnico» que está por encima de todos ellos y tiene una visión conjunta y única del proceso edificatorio, teniendo presente desde el principio el edificio o producto final y encaminando todas las fases del proceso a la mejor obtención del resultado.

Según esta idea, se ha comparado a menudo al arquitecto con el director de orquesta que conoce todos los instrumentos que intervienen y los dirige y coordina con el objeto de obtener, en el «proceso musical», una buena interpretación $y$, por tanto, una obra bella, bien construida.

En este mismo sentido, se puede extender la comparación al compositor musical, con las mismas exi- 

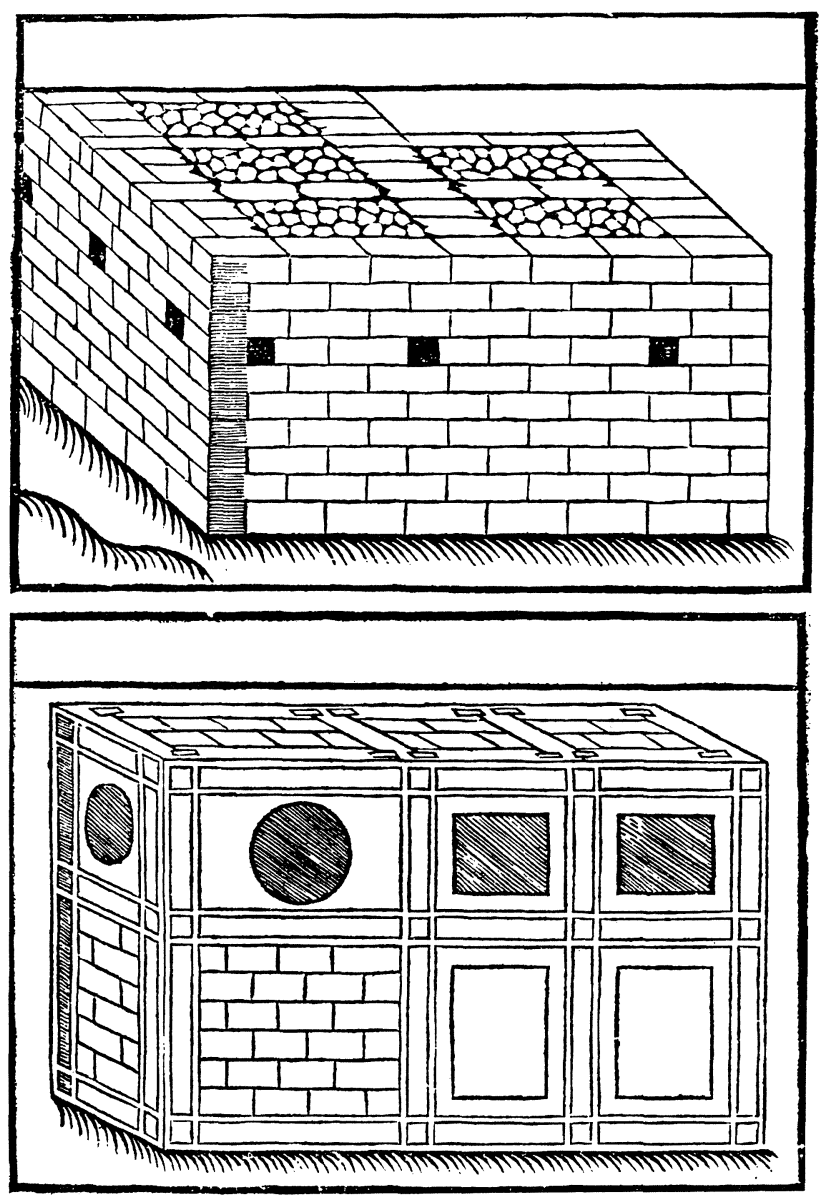

De Architectura

Marco Vitrubio Polión. Libro segundo

Versión de Miguel de Urrea. Madrid, 1582

Edición Facsimil, Albatros Ediciones, 1978

gencias de conocimiento de instrumentos y coordinación de ellos en el proceso de composición, asemejando la labor de éste a la del proyectista y la de aquél al director de obra.

Para llevar adelante su cometido de un modo correcto y eficaz, al igual que el director de orquesta o el compositor conocen el manejo de los diversos instrumentos, el arquitecto, como coordinador y super-técnico, debe conocer, e incluso dominar, la labor de los diversos técnicos que intervienen en el proceso y, por tanto, la base y el método de sus técnicas; y no sólo la de los proyectistas, aunque sea el aspecto en el que se hace más hincapié en su actual formación, sino, además, la de los fabricantes, constructores, etcétera.

A este respecto, y para apoyar históricamente esta definición, quiero traer a colación dos citas que considero suficientemente ilustrativas.

Vitrubio, en su libro primero (versión al castellano de Miguel de Urrea, de 1582) (1), dice:

"La Arquitectura es una ciencia adornada de muchas disciplinas y varia erudición, la cual juzga y aprueba todas las obras de las otras artes. Esta ciencia nace de fábrica y razón. La fábrica es una razón continuada y muy trillada del uso, la cual es perfeccionada con las manos, y es necesaria la materia apropiada a propósito de la formación. La razón es la que puede mostrar y explicar las cosas compuestas con diligencia y proporción. De manera que aquellos arquitectos que sin letras contendieron, aunque ellos fueron ejercitados con sus manos, no pudieron hacer que tuviesen autoridad sus trabajos. Y los que se confiaron en sola razón y letras alcanzaron la sombra del arte, pero no la verdadera ciencia, empero los que aprendieron lo uno y lo otro, como hombres adornados de todas armas, más presto alcanzaron con autoridad lo que procuraron. Porque en todas las cosas se contienen estas dos, y mayormente en la Arquitectura, que es lo significado y lo que significa. Lo significado es la cosa propuesta, de la cual se ha dicho, más la demostración explicada con razones de doctrina demuestra lo que significa. Por lo cual, es visto, que aquellos que profesan ser arquitectos han de ser ejercitados en lo uno y en lo otro, de manera que conviene ser ingeniosos y fáciles para aprender la ciencia, porque ni la ciencia sin ingenio, ni el ingenio sin la ciencia pueden hacer perfecto artífice. Conviene pues que el arquitecto sea letrado en dibujo y traza, y que sea entendido en la geometría, y que no ignore la perspectiva, y que sea instructo y enseñado en la aritméti$c a, y$ que haya visto muchas historias, y que haya oído los filósofos con diligencia, y que sepa música, y que no sea ignorante de la medicina, y que conozca las respuestas de los letrados y que sea astrólogo y conozca los movimientos y razones del cielo».

Sebastián Serlio, el arquitecto boloñés del Renacimiento, llega hasta el nivel de la anécdota para hacer hincapié en esa necesidad de conocimiento universal por parte del arquitecto tocando un tema, más específico, cual es el de la decoración pictórica final. Así, nos dice en su libro cuarto, capítulo XI (traducido al castellano por Francisco Villalpando en 1552) (2):

«Por no dexar ninguna suerte de los ornamentos de los edificios, ansi de la pintura como de las otras cosas en que yo no de alguna regla. Digo, que el arquitecto no solamente debe ser un curioso de los ornamentos que han de ser de piedra y marmol, pero también lo debe ser en obra y pintura de pincel para adornar las paredes y otras partes de los edificios, y principalmente le conviene ser el mismo ordenador de todo como superior de todo lo que se haya de hazer en las obras: porque sino lo es, podría topar con algunos pintores tan presuntuosos en las palabras $y$ en saber estimarse, cuanto en las obras de poco juicio: los cuales no han tenido ni tienen respecto a más de mostrar las diferencias de los colores, sin consideración a otra cosa ninguna, y con esto muchas vezes han corrompido la orden que 
se debe tener en las tales cosas, no teniendo cuenta de poner la pintura en su lugar, y los coloridos donde son necesarios».

En definitiva, el arquitecto, no sólo de hoy, sino a lo largo de toda la historia de la Arquitectura, ha tenido, y debe tener, un carácter de coordinador y director de técnicos, de artífices, cuyas técnicas forzosamente debe conocer, y una formación lo más universal posible, dentro de su campo, con objeto de no perder la visión de conjunto de un proceso edificatorio, desde su proyecto hasta su ejecución, $y$ de tener como centro de su actividad al hombre, para quien van destinados, en definitiva, los edificios que proyecta y construye.

\section{LA TECNOLOGIA CONSTRUCTIVA COMO ARTE Y CIENCIA DE CONSTRUIR}

Buscando de nuevo en el diccionario de la Real Academia Española de la Lengua, encontramos la definición de tecnología como «conjunto de los conocimientos propios de un oficio mecánico o arte industrial».

También nos interesa el significado de la palabra técnica que a menudo se confunde en el lenguaje ordinario ( $y$, a veces, en el culto) con tecnología.

El diccionario define técnica como «conjunto de procedimientos y recursos de que se sirve una ciencia o un arte».

Uniendo las dos podríamos decir que la tecnología supone el estudio del conjunto de técnicas que intervienen en un proceso artístico o industrial.

Por otra parte, Melvin Kranzberg y Carrol W. Pursell, en su obra «Historia de la Tecnología» (3), indican que:

«En su explicación más sencilla, la tecnología consiste en los esfuerzos del hombre para enfrentarse a su entorno físico - tanto el que aporta la Naturaleza como el creado por los propios logros tecnológicos del hombre, por ejemplo, las ciudades - y sus intentos para dominar o controlar ese entorno por medio de su imaginación e ingenio en la utilización de los recursos disponibles».

Ese, podríamos decir, es el objetivo de las diversas técnicas. La tecnología es el estudio y desarrollo de todas ellas. En este sentido, para hablar de tecnología necesitamos de un proceso determinado donde se trate de obtener algo, un producto. Entonces, podremos hablar de la «tecnología del proceso».

En edificación se utilizan una serie de técnicas para construir el edificio. Su conjunto constituye lo que podríamos entender como «tecnología del proceso constructivo», "tecnología constructiva», es decir, el

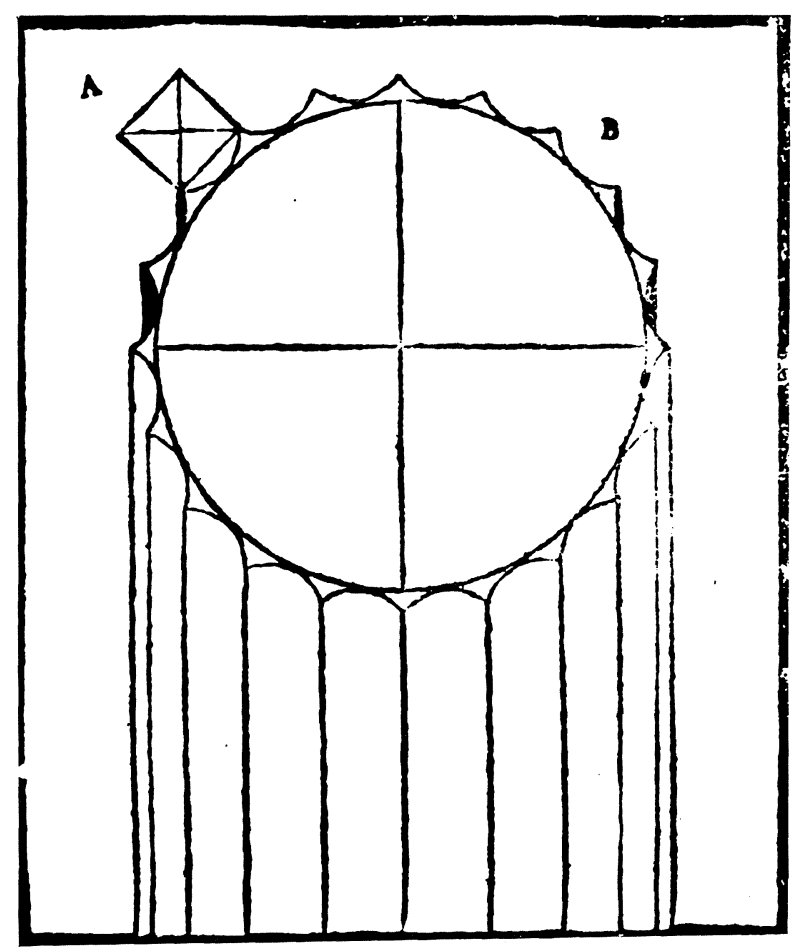

De Architectura

Marco Vitrubio Polión. Libro quarto

Versión de Miguel de Urrea. Madrid, 1582

Edición Facsimil, Albatros Ediciones, 1978

análisis del conjunto de técnicas constructivas que intervienen en el arte de edificar.

Los mismos autores anteriores afirman en el libro mencionado:

"Y es que en realidad vivimos en una "Era Tecnológica". No se llama así porque todos los hombres sean ingenieros, ni tampoco porque todos los hombres comprendan la tecnología, sino porque cada vez somos más conscientes de que la tecnología se ha convertido en una gran fuerza desbaratadora, al mismo tiempo que creativa del siglo $\mathrm{XX}$.

Podemos, pues, acercarnos al estudio de la tecnología constructiva como simples técnicos estudiosos, fría y científicamente, de los diversos métodos, o podemos hacerlo conscientes, por una parte, de la carga creativa que llevan consigo las técnicas $y$, por otra, del objetivo humanista de su uso, encaminado, como apuntan Kranzberg y Pursell, al bienestar del hombre.

Para el arquitecto, considero imprescindibles esos últimos enfoques que le obligan a no parar en un simple estudio de las técnicas de construcción y profundizar, sin embargo, en los orígenes de cada una de ellas, sus bases científicas y su posterior desarrollo y mejora. No en balde, etimológicamente, la palabra tecnología viene de los vocablos griegos "tectun» (arte) y "logos» (estudio), es decir, estudio del arte de un proceso de producción. 


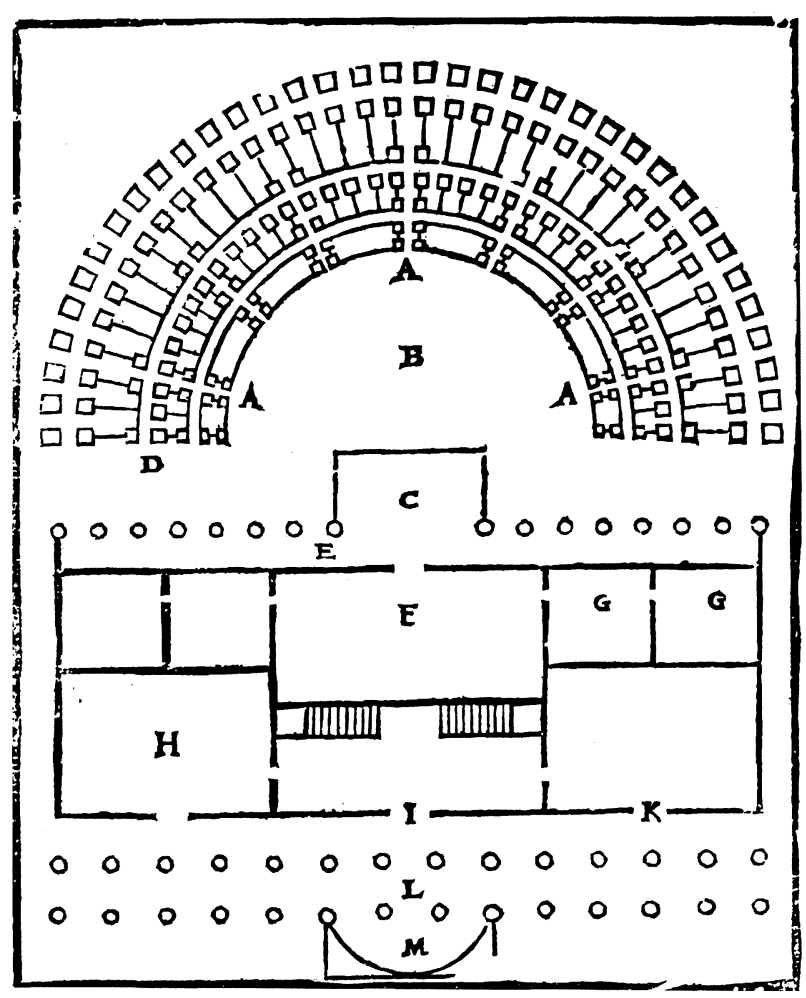

De Architectura

Marco Vitrubio Polión. Libro quinto

Versión de Miguel de Urrea. Madrid, 1582

Edición Facsímil, Albatros Ediciones, 1978

Cabe mencionar, además, un hecho histórico, en relación con el enfoque de las diversas técnicas, que nos interesa sobremanera desde el punto de vista de su estudio y que ya hemos apuntado en la introducción de este trabajo. La separación tradicional entre técnica y ciencia. Kranzberg y Pursell consideran que la ciencia es un intento por parte del hombre para "comprender» el mundo físico, mientras la técnica es un intento para «controlarlo». Sin embargo, la historia nos enseña que desde las civilizaciones primitivas la ciencia ha estado en manos de los dominantes y de los religiosos, mientras que la técnica ha estado en manos de los artesanos, de los ingeniosos. En realidad, hasta la revolución industrial no existe un verdadero acercamiento entre los dos campos y es entonces cuando el desarrollo tecnológico es mayor, pues la tecnología empieza a usar bases científicas y la ciencia aprovecha los logros de la aplicación tecnológica.

Por otra parte, según James Dick, director de «Building Research Establishment», la construcción es una «tecnología con base científica de reciente desarrollo». Sin embargo, la peculiaridad de la historia de la construcción moderna es el paso, en lenta evolución, de una "tecnología empírica a una ciencia aplicada» que es como, en definitiva, nos interesa contemplarla. D. A. G. Reid afirma en su libro «Principios de Construcción» (4):

"La construcción se ha desarrollado como una tecnología empírica; las técnicas estaban evolu- cionando por un proceso de ensayos y errores sucesivos y sólo durante la última centuria y media ha aparecido una sostenida inclinación a cambiar este proceder. Lo mismo podría decirse de muchas de las actividades humanas clasificadas como artes útiles. El método de ensayo-error con el que desarrollaban las tecnologías empíricas, es necesariamente lento en su evolución y éste es particularmente el caso de la construcción, donde es necesario esperar un período de tiempo, que a menudo es de varios años, antes de poder juzgar los resultados de una nueva técnica o material. Además, en el caso que nos ocupa, la experimentación que nos satisface se basa en el propio hecho de construir y como consecuencia de ello los errores tienden a ser caros $y$, a veces, peligrosos para la vida humana».

En definitiva, es importante acercarnos al estudio de la tecnología constructiva con dos enfoques paralelos: De un modo científico, por un lado, con aplicaciones técnicas en la construcción, o como una tecnología basada en datos científicos. Por otro, como indica la etimología de su palabra, como un estudio del arte de edificar $y$, por tanto, con toda la capacidad creativa que ello implica. De hecho, hemos visto que cuando en 1844 se reorganizaron los estudios de las Tres Nobles Artes en nuestro país, el estudio de la Arquitectura se dividió en dos ramas distintas, la de la Bella Arquitectura, por un lado, y la del Arte o Ciencia de Construir, por otro.

\section{La física de la construcción}

Esta dicotomía histórica entre técnica (arte) y ciencia, se mantuvo claramente hasta bien entrado el siglo XVIII en que, con la revolución industrial se facilitó la aproximación entre las dos ramas. Gautier, ingeniero francés de principios del siglo XVIII, en su libro sobre puentes, editado en 1716 , se quejaba de que "les savants» (los sabios) no se interesaban por la mecánica de los arcos, mientras que los arquitectos se dejaban absorber por la estética del diseño.

Quizá la Arquitectura ha sido la profesión en donde más ha costado establecer la colaboración entre la ciencia y la tecnología y donde, probablemente, hoy todavía es muy reducida. Naturalmente esa colaboración debe darse fundamentalmente en el aspecto tecnológico de la Arquitectura, es decir, en su técnica constructiva. De tal modo que, en mi opinión, la tecnología constructiva (la construcción) debe considerarse como una ciencia aplicada o, cuanto menos, las técnicas constructivas deben tener una férrea base científica, y esta ciencia en la que se base debe ser, fundamentalmente, la física.

Por otra parte, son evidentes las interacciones físicoquímicas que se producen, tanto entre los diversos materiales y elementos componentes de una cons- 
trucción, como entre el edificio y el medio que le rodea. Es indudable que este tipo de interacciones están dominadas por unas leyes físicas y que ellas constituyen los datos científicos básicos para la selección de las características exigibles a los materiales y elementos a utilizar.

Por último, en mi opinión, el diseño constructivo, como parte del diseño global de un edificio, tiene por misión resolver un problema que se presenta como geométrico, físico y mecánico; tres aspectos ordenados por leyes físicas. En resumen, considero que la tecnología constructiva debe enfocarse como una ciencia física aplicada.

Gerard Blachère, al que considero uno de los padres de la construcción como ciencia física, lo expresa claramente en su libro "Saber construir» (8), de cuyo capítulo primero merecen destacarse algunos párrafos:

«Recapitulemos: exigencias del ocupante, incluso exigencias económicas, a saber, exigencias de habilidad, de durabilidad y de economía, exigencias de la colectividad, datos naturales, datos exteriores impuestos, condiciones normales de ocupación, he aquí el conjunto de datos para plantear el problema. Edificar es resolver ese problema.

Ahora sustentemos el criterio de que se puede y, por consiguiente, se debe resolver con base científica y ya no empíricamente. Esto equivale a decir que disponemos de los conocimientos científicos suficientes para resolver el problema que acabamos de plantear, es decir, que la acústica y la terminología aplicada a los edificios y asimismo la iluminación, la resistencia de materiales, la sociología y la economía están ya bastante avanzadas para lograr la susodicha solución».

Y no sólo en los libros se capta esta nueva preocupación sino, incluso, en la normativa oficial. Concretamente, la última normativa española sobre edificación refleja la preocupación por esta interacción física y, al igual que en otros países, se orienta a especificar condiciones para una adecuada interacción. Así, habla de condiciones térmicas, condiciones acústicas, etc., variando el antiguo criterio de normativa técnica en la que se limitaban soluciones concretas y no condiciones de funcionamiento.

Pero también los propios arquitectos en su práctica profesional crecen en esa preocupación y consideran en su diseño el funcionamiento físico del edificio, aunque quizá todavía no al nivel que sería de desear. A este respecto Edward Allen afirma en su libro "Cómo funciona un edificio» (9):

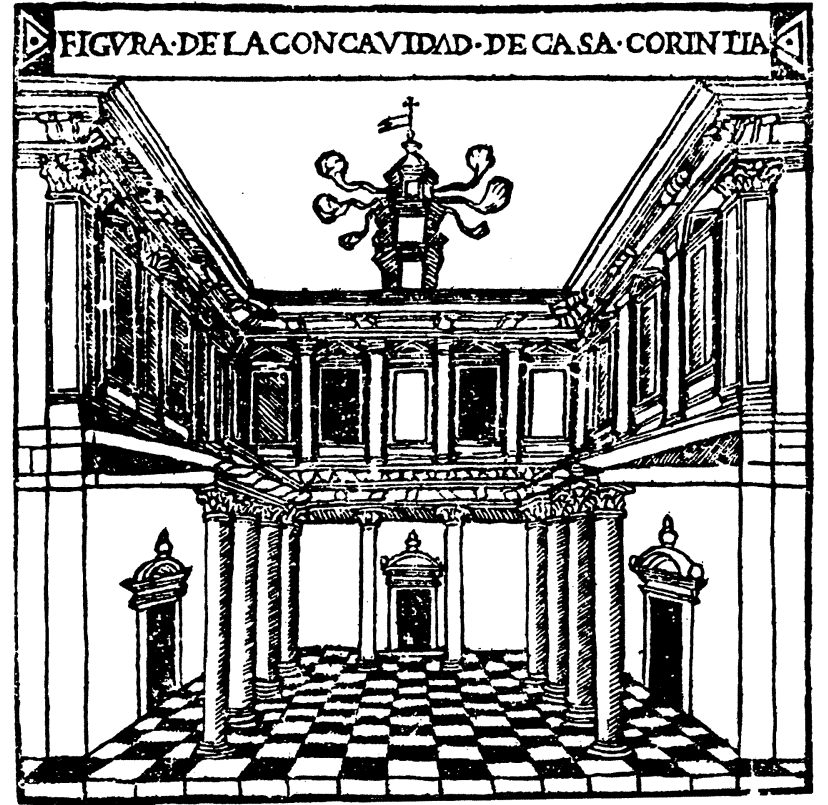

De Architectura

Marco Vitrubio Polión. Libro sexto

Versión de Miguel de Urrea. Madrid, 1582

Edición Facsimil, Albatros Ediciones, 1978

«Hay unas reglas naturales para la Arquitectura, y unas reglas no limitadas por el tiempo, el lugar o el estilo, más duraderas que las reglas dóricas o jónicas de los antiguos griegos, y más metódicas que el estilo contemporáneo más limpio. Son las reglas de la función física, de las maneras que tienen los edificios de comportarse. Cuando se las entiende como un conjunto tienen una elegancia y una utilidad que si no, no pueden ser apreciadas y explotadas».

\section{LA TECNOLOGIA CONSTRUCTIVA EN LA FORMACION DEL ARQUITECTO}

Históricamente, la tecnología constructiva ha estado asociada siempre a la Arquitectura de tal modo que los arquitectos, hasta la edad contemporánea, han sido una especie de artistas-constructores (artífices) al servicio de los señores, pues como arte se consideraba a la técnica de construir (el arte de construir). De ese modo, en la formación de los arquitectos se tenía presente la enseñanza de las diversas técnicas y la idea del arquitecto iba aparejada, no sólo a la del gran artista, sino también a la del matemático, a la del científico, o a la del técnico. Así nos lo prueban todos los tratados sobre Arquitectura y Construcción, desde los diez libros de Vitrubio, ya mencionados, y prácticamente todos los que se conservan de la época renacentista (quizá la más fértil en este tipo de trabajos), hasta los más modernos que forman parte de la historia.

Y no sólo a la técnica, sino también al saber científico, se le suponía como formando parte del conocimiento de los arquitectos, tanto que por ellos se 


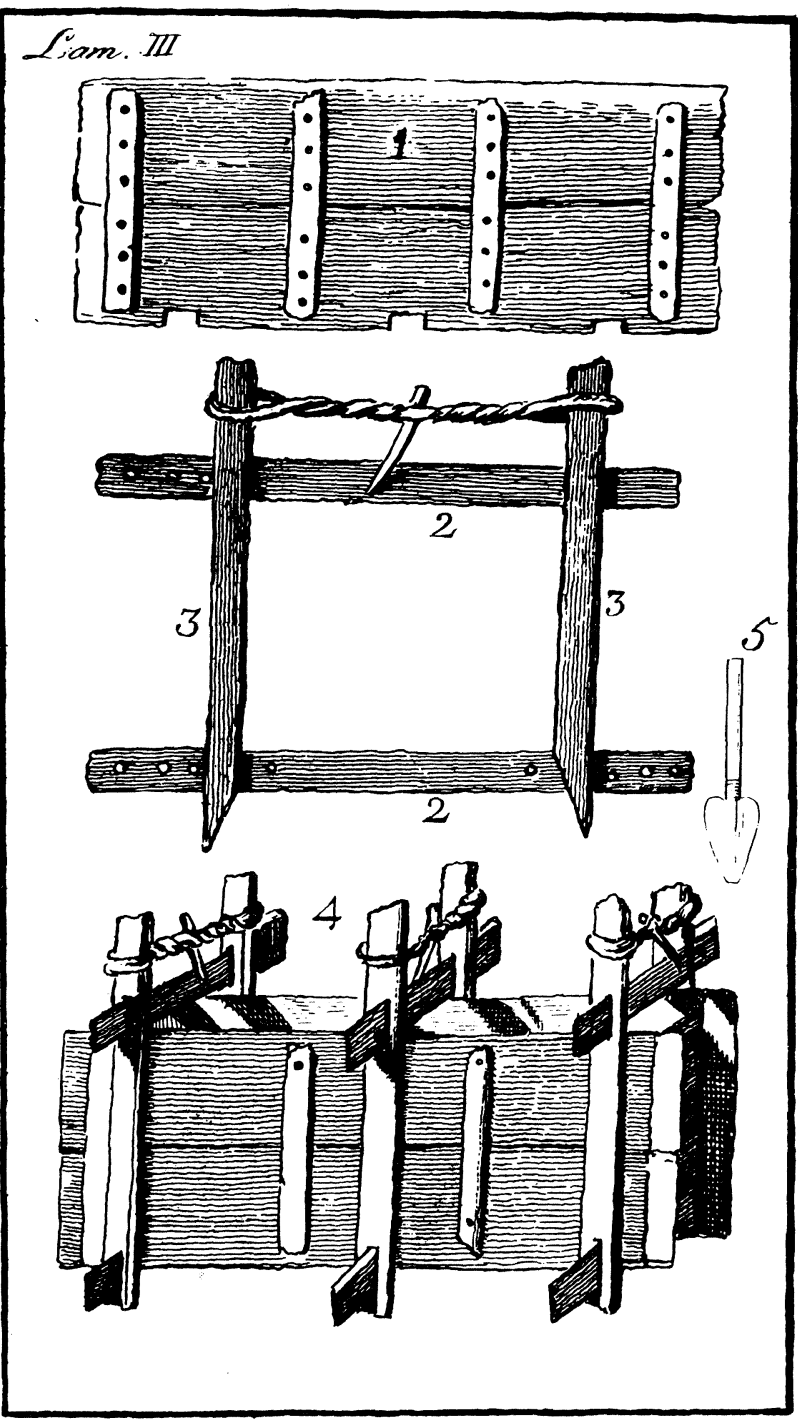

Lám. III Fol. 25

ARTE DE ALBAÑILERIA

Juan de Villanueva

Madrid, 1827

Edición Facsímil de Ed. Velázquez. Madrid, 1977

podrían clasificar y distinguir unos de otros. Juan de Torija, arquitecto y tratadista de construcción del Siglo de Oro español, afirma en su «Breve tratado de todo género de bóvedas», editado en Madrid en 1661 (5):

«Lo científico se adquiere, no por lo verboso, ni por solicitud o multitud de amigos, sino por el develo y execución, convirtiendo las noches en días, por lo despierto en los libros, procurando saber de los grandes maestros la ciencia que se profesa».

La idea básica es que el quehacer de un arquitecto es eminentemente práctico $y$, por tanto, sus conocimientos deben estar impregnados por este saber técnico que va aparejado con el aspecto práctico.

Manuel Fornés y Gurrea, arquitecto, director de Ar- quitectura de la Academia de San Carlos de Valencia y tratadista eminente de Arquitectura del tardoneoclasicismo, indica en la introducción a su libro "Observaciones sobre la práctica del Arte de Edificar» (6), recientemente reeditado:

"Tales son las consideraciones que he tenido a la vista al escribir las «Observaciones sobre la práctica del Arte de Edificar» que a fin de hacer con ellas más palpable aquella verdad tan demostrada, que la teoría sin los conocimientos prácticos, sólo sirven para alucinar la ignorancia y estupidez de los que con el solo caudal de principios y conocimientos superficiales hacen invertir sumas cuantiosas, sin más seguridad para el diseño, que una esperanza, la más veces fallida».

Y más adelante, en su "Tratado primero", concreta esta afirmación refiriéndose a la necesidad de algún tipo de «pliego de condiciones» en los proyectos:

«Es decir, que además del diseño y explicación de lo que contiene debe acompañar una exacta y prolija demostración de cómo han de entenderse y efectuarse, e igualmente las especies de materiales que hayan de entrar en su elaboración, conforme a las reglas de buena edificación».

Argumento, por demás, totalmente actual.

Pero esta aceptación del arquitecto como técnico o, mejor dicho, con los suficientes conocimientos técnicos, tan entroncada en la cultura a lo largo de toda la historia, empezó a zozobrar cuando, a partir de la revolución industrial, se incrementa enormemente el dominio de la tecnología como concepto y se multiplican las técnicas en uso, tanto que cada vez se hace más difícil que un técnico, un artífice, sea entendido en cualquier técnica y las domine a todas. Fue el momento en que aparecieron los ingenieros (ingeniosos) como profesión.

Ya antiguamente, y sobre todo a partir del Renacimiento italiano, se alzaban voces a favor del aspecto únicamente artístico de la Arquitectura aunque nunca la podían desligar de la tecnología constructiva.

A partir del siglo XIX, las fortificaciones del momento supusieron la aparición de ingenieros («ingeniator») militares dada la complicación de las mismas.

En España podríamos decir que es a partir de 1844 en que se reorganizan los estudios de las tres Nobles Artes, diez años después de la creación de la primera Escuela de Ingenieros de Caminos, Canales y Puertos, cuando se oficializa el concepto artístico de la Arquitectura dividiéndose sus estudios en las dos ramas ya mencionadas, la Bella Arquitectura y el Arte o Ciencia de Construir. (Obsérvese 
que la tecnología constructiva, aunque se separa de la "Bella Arquitectura», se sigue estudiando como parte de ella).

Sin embargo, a partir de entonces, como señala el crítico Bonet Correa (7):

"La duplicidad de títulos - ingeniero, arquitecto- generó la dicotomía constructor y diseñador. La polémica ingeniero-arquitecto no sólo tuvo carácter teórico, sino también general y de competencia. En torno a ello se debatieron problemas esenciales como los de clasicismo y modernidad, tradición y novedad, historicismo y eclecticismo. La división entre obras públicas estatales y clientela particular, también entre edificios públicos y propiedades de sociedades o individuos civiles».

Polémica que se mantiene viva en la actualidad, quizá por no estar bien resuelta la distribución de responsabilidades. Y no sólo a nivel profesional sino también a nivel universitario donde se discute la oportunidad de incluir la tecnología constructiva en la enseñanza actual de la Arquitectura; polémica que también se mantiene a escala internacional, con una evidente división de opiniones, como se ha puesto de manifiesto en las últimas reuniones de la AEEA (Asociación Europea para la Enseñanza de la Arquitectura).

Considero que hay argumentos suficientes para defender la formación tecnológica del arquitecto, no sólo históricos (como hemos visto), sino además funcionales $y$, en nuestro caso, legales; veamos: Por una parte, es evidente que el objeto final de un proyecto de edificación es que se pueda construir y que la idea plasmada en el mismo se convierta en realidad. Tiene que existir, por tanto, una estrecha coordinación entre proyecto y posibilidad de ejecución o, lo que es lo mismo, unas consideraciones constructivas importantes durante la fase de proyecto. No quiere ello decir que !a tecnología constructiva condicione el proyecto, pero sí que aquél se realice pensando en cómo se va a construir, bien utilizando las técnicas y sistemas del mercado, bien definiendo y diseñando las técnicas y los sistemas necesarios para su construcción.

Al fin y al cabo, en el proyecto arquitectónico debemos considerar básicamente tres componentes del diseño: el diseño funcional, el diseño estético y el diseño constructivo, y los tres tienen que unificarse en uno solo, pues de un proyecto se trata. Los tres componentes tienen igual importancia y exigen los conocimientos suficientes, por parte del proyectista, de tal modo que un proyecto pensado sólo desde uno cualquiera de los tres aspectos no puede considerarse completo.

En definitiva, necesitamos, o bien una intima colaboración entre diseñador y especialista en construcción durante todo el proceso, o bien que el di- señador (arquitecto) tenga los conocimientos suficientes sobre construcción para desarollar un proyecto construible dentro de un margen económico determinado. De hecho, cuanto más profundos sean sus conocimientos sobre técnicas y sistemas constructivos, menores serán los inconvenientes que encuentre en el diseño y, por tanto, mayor será su libertad para proyectar.

Cabe afirmar que cualquier línea que compone el diseño de un proyecto representa un elemento constructivo - un componente- que formará parte de un sistema y que se deberá ejecutar con una técnica determinada. Técnicas, sistemas y elementos constructivos que deberán formar parte del conocimiento científico del proyectista.

Pero el arquitecto no solamente proyecta. También dirige la construcción de un edificio ya proyectado por él mismo o por otro colega, y la legislación española le hace especialmente responsable de los problemas técnicos que puedan surgir en una edificación.

Por tanto, no se trata de formar sólo arquitectos proyectistas. Debemos formar también arquitectos edificadores o directores de obra. $Y$ en este segundo campo no hay duda de la necesidad de una formación técnica, al margen de unos convenientes conocimientos sobre organización y economía.

\section{LA ARQUITECTURA COMO «ARTE FUNCIONAL»}

Aceptada la idea de la Arquitectúra como arte funcional mencionada al principio del trabajo, y después de las consideraciones anteriores, conviene analizar los diferentes aspectos de esa "funcionalidad» para conocer el modo cómo la tecnología constructiva puede facilitar su logro.

Tres son, en mi opinión, los aspectos básicos de la funcionalidad arquitectónica: La integridad, la habitabilidad y la estética (Vitrubio hablaba de "Firmirtas», «Utilitas»y «Venustas»).

\section{A) Integridad}

La integridad se refiere a la permanencia del edificio. Si tiene que ser vivido, exige un mínimo de duración. No es concebible una arquitectura efímera aunque sí lo es una arquitectura temporal. Se tiene que partir de un tiempo mínimo de duración y diseñarla para que lo cumpla en las mejores condiciones, es decir, sin degradaciones ni funcionales ni estéticas.

Podemos distinguir dos tipos de integridad:

A-1. Integridad mecánica, que comporta la exigencia de un sorporte, desde el más elemental, que da cubierta a un espacio habitable, hasta el más com- 


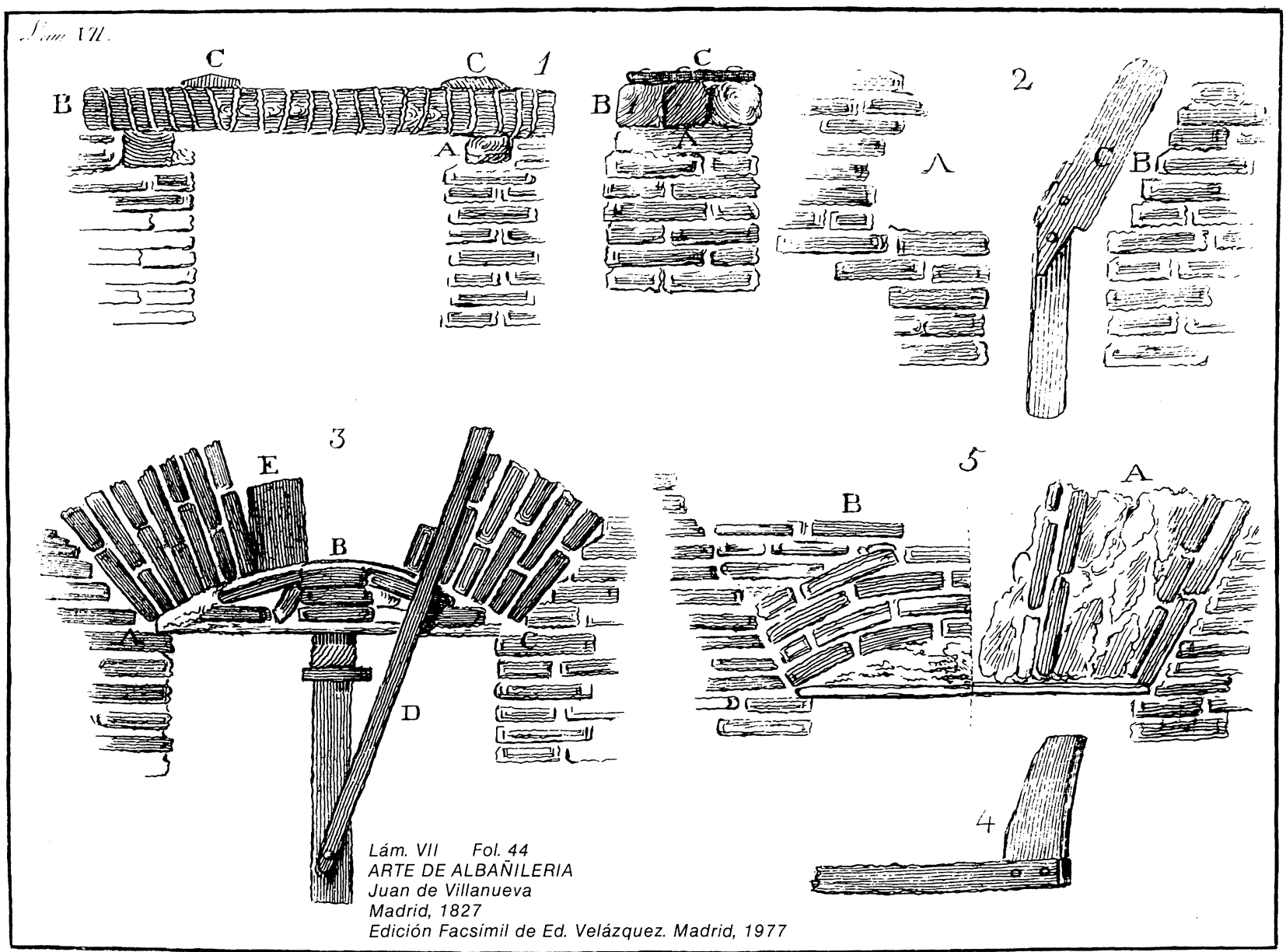

plejo, que permite obtener las formas y los espacios más arriesgados y que, en cualquier caso, tiene que conferir a la arquitectura la seguridad de su estabilidad.

Exigencia, pues, de una estructura en el edificio, diseñada en consonancia con su funcionalidad, aprovechando la creatividad de las ciencias de la mecánica y de la estática.

A-2. Integridad física; supone la suficiente resistencia ante los agentes externos, más o menos agresivos a lo largo del tiempo, para conferir a la arquitectura la durabilidad físico-química que le da la permanencia funcional y formal durante su vida útil.

Estos agentes externos son muchos y muy variados, por lo que la tecnología en este campo tiene que desplegar sus «artes». Mencionemos los más importantes.

Como Agentes Naturales actuando sobre los cerramientos (fachadas y cubiertas) de nuestros edificios, debemos considerar los elementos básicos de la Naturaleza:

- El agua, en sus estados sólidos (hielo que destruye, nieve que pesa), liquido (lluvia, agua infiltra- da, agua embalsada que tratan de penetrar) y gaseoso (vapor de agua en suspensión que se filtra).

- El sol, con sus tres zonas del espectro de radiaciones: la infrarroja (temperatura), la luminosa (soleamiento) y la ultravioleta (efectos químicos).

- El viento, con su efecto de carga dinámica y de vehículo de partículas en suspensión que erosionan superficies o que producen lavados desiguales en las fachadas $y$, por tanto, suciedades efectivas.

- Los animales, sobre todo los insectos, que no sólo atacan algunos materiales de construcción, sino que, además, son molestos en el interior de los edificios.

- Las plantas, que actúan básicamente desde el subsuelo por medio de sus raíces.

Como Agentes Artificiales consideramos, por un lado, al hombre, cuando trata de atacar la propiedad ajena, $y$, por otro, los efectos de sus actuaciones, principalmente el fuego, pernicioso cuando no se controla, la contaminación atmosférica, tanto la industrial como la urbana de tráfico y calefacciones, 
que contribuye, en buena medida, al ataque químico de las fachadas $y$, sobre todo, a su ensuciamiento, y, por último, el ruido, que dificulta la adecuada obtención del confort acústico.

Para hacer frente a estos y otros agentes requeriremos a la tecnología constructiva que «cree» las soluciones adecuadas para los cerramientos de nuestros edificios y las utilizaremos en nuestro proceso de diseño como solución técnica que hace viable la solución funcional de nuestra arquitectura.

\section{B) Habitabilidad}

Constituye el aspecto básico de la funcionalidad en la Arquitectura y su objetivo principal; habitabilidad que deberá adecuarse, en cada caso, al fin funcional del edificio, ya sea residencia, oficina, industria, comercio, etc., y que, en cualquier caso, permitirá que la arquitectura pueda ser vivida en las condiciones más adecuadas.

Distinguimos, asimismo, dos aspectos a cubrir en el diseño para alcanzar la habitabilidad.

B-1. En primer lugar, deberemos obtener una adecuada distribución interior de los espacios, lo que implica, por un lado, el conseguir que cada espacio habitable tenga la forma y tamaño más adecuados para su función $y$, por otro, que la relación entre ellos sea la más funcional; aspectos estos primordiales en el proceso de diseño, pero no únicos.

En efecto, esto supone un primer paso en la utilidad del edificio para ser habitable, en el que la tecnología constructiva no tiene un papel fundamental, pero hay que completarlo con un segundo aspecto igualmente importante.

B-2. La aptitud físico-química de la envoltura de cada espacio y de su ambiente para la habitabilidad interior.

Un espacio no es habitable sólo por su forma y tamaño, sino, además, por su confort ambiental, confort que queda definido por los datos de temperatura, humedad relativa, ventilación, nivel lumínico y nivel acústico, fundamentalmente.

Un diseño arquitectónico no es completo si no tiene en cuenta, y resuelve, estos aspectos. Su solución puede alcanzarse utilizando los elementos naturales exteriores o empleando medios mecánicos, o por una combinación de ambos. En cualquiera de los casos, la solución deberá contemplar, por lo menos:

- la orientación de las fachadas exteriores del espacio habitable;

- el soleamiento efectivo que condicionará el tamaño y forma de los huecos, así como su protección;
- la iluminación natural en íntima relación con el punto anterior;

- la ventilación natural, que además condiciona la practicabilidad de las ventanas;

- el funcionamiento higrotérmico de los cerramientos, con análisis, tanto del gradiente de temperaturas, como del de humedad a través de ellos, localizando y evitando posibles condensaciones.

En todo ello juega un papel fundamental la Tecnología, que tendrá que "crear» y comercializar materiales y sistemas constructivos de cerramiento con el máximo coeficiente de aislamiento térmico y el mejor aprovechameinto de la inercia térmica de los materiales según su colocación relativa, a materiales transparentes y translúcidos que puedan tamizar adecuadamente las radiaciones solares, sin eliminar la necesaria visibilidad a su través, o materiales y sistemas de revestimiento y protección exterior capaces de establecer una barrera efectiva a los agentes no deseables en un momento determinado, sean naturales (agua, sol, viento, animales) o artificiales (ataque humano, fuego, contaminación), materiales y sistemas que, por otra parte, sean permanentes, de fácil mantenimiento y hagan posible la solución más adecuadamente habitable para cada arquitectura.

\section{C) Estética}

La estética en la Arquitectura répresenta un aspecto muy importante de su funcionamiento, pero no el único. De hecho, a menudo se piensa que el estético es el objetivo básico de este arte y el profano lo considera como su característica fundamental. Nada más lejos de la realidad, según estamos viendo. No obstante, es un aspecto que no podemos perder de vista y que, junto con los dos anteriores, completa la funcionalidad de la Arquitectura.

Cualquier arquitectura no puede ser sólo permanente o habitable o estética, sino que tiene que ser las tres cosas a la vez. Es el trípode en el que se apoya su funcionalidad.

En cualquier caso, cabe considerar dos tipos de estética en la Arquitectura: la exterior y la interior.

C-1. La estética exterior determina el aspecto visual de un edificio y, como tal, permite la expresión de su funcionalidad. Pero además, la fachada de una arquitectura es la pared de un espacio exterior y define, por tanto, parte del entorno en que se asienta. Esto resulta particularmente importante en el caso del entorno urbano, que puede considerarse como absolutamente definido por la arquitectura que lo rodea. Sin embargo, buscar una expresión formal que se base exclusivamente en una composición de formas y volúmenes con un objetivo pura- 
mente estético, puede alejarse de la raíz de la Arquitectura y adentrarse peligrosamente en el campo de la Escultura, arte que de por sí tiene su dominio, su origen y sus objetivos.

C-2. La estética interior afecta directamente a los usuarios de cada arquitectura y tiene una íntima interdependencia con la habitabilidad. Cada espacio habitable, para serlo adecuadamente, para que el usuario se sienta a gusto en él y pueda desarrollar su actividad de la mejor manera posible, debe estar proyectado y ejecutado contemplando y resolviendo su estética; la de su forma y volumen totales, la de su iluminación, la de la decoración de sus superficies interiores (paredes, techo y suelo) incluso la de su mobiliario; estética que, inevitablemente, dependerá de la funcionalidad del local para, a su vez, completarla adecuadamente.

En fin, para que la ejecución de una arquitectura logre los objetivos estéticos que su funcionalidad requiere, tanto en sus espacios interiores como en su entorno, también hará falta echar mano de la Tecnología y de su capacidad creativa y utilizar las técnicas adecuadas para que los sistemas y materiales constructivos respondan a las intenciones del proyecto.

Esa Tecnología que sea capaz de crear las técnicas que resuelvan adecuadamente los tres aspectos de la funcionalidad analizados - integridad, habitabilidad y estética - es la que constituye el Arte, Técnica o Ciencia de la Construcción que complementa la Arquitectura como Bella Arte.
En definitiva, podemos hacer hincapié, una vez más, en la importancia de la funcionalidad como característica básica de la Arquitectura y en la consideración de la Tecnología Constructiva y su creatividad potencial (Arte de Construir) como soporte básico de esa bella arte. Importancia e interdependencia que deben hacerse patentes, por un lado, en la formación universitaria de los arquitectos (léase Escuelas de Arquitectura) y, por otro, en la actuación profesional de esos arquitectos (artistas y artífices a la vez) cuya responsabilidad ante la sociedad les exige una permanente creatividad funcional, $y$, por tanto, tecnológica.

\section{BIBLIOGRAFIA}

1. De Architectura. M. Vitrubio Polión. (Traducción de Miguel de Urrea, 1582). Ed. Albatros (M. ${ }^{\circ}$ de Educación y Ciencia, 1978).

2. Tercero y cuarto libro de Architectura. Sebastián Serlio Boloñés. (Traducción de Francisco Villalpando, 1552). Ed. Albatros (M. ${ }^{\circ}$ de Educación y Ciencia, 1977).

3. Historia de la Tecnología. Melvin Kranzbeg y Carroll Pursell, Jr. Ed. Gustavo Gili, 1980.

4. Principios de Construcción. D. A. G. Reid. Ed. Gustavo Gili, 1980.

5. Breve tratado de todo tipo de bóvedas. Juan de Torija. Ed. Albatros (M. ${ }^{\circ}$ de Educación y Ciencia, 1981).

6. Observaciones sobre la práctica del arte de edificar. Manuel Fornes y Gurrea.

7. Prólogo del libro anterior.

8. Saber construir. Gerard Blachère. Editores Técnicos Asociados, S. A., 1966.

9. Cómo funciona un edificio. Principios elementales. Edward Allen. Ed. Gustavo Gili, 1981.

\section{publicación del i.e.t.c.c.}

\section{ACUEDUCTOS ROMANOS EN ESPAÑA Carlos Fernández Casado Prof. Dr. Ing. de Caminos, Canales y Puertos}

\begin{abstract}
Esta publicación se compone de una serie de articulos, publicados en la Revista "Informes de la Construcción", en los cuales se hace un análisis de los acueductos romanos que existen en España y el balance de las condiciones de conservación en que se encuentra cada uno de ellos, incluyendo referencias históricas y literarias. Se ha ilustrado con la reproducción de la valiosa documentación gráfica que posee el prestigioso autor.
\end{abstract}

Un volumen encuadernado en couché, a dos colores, de $21 \times 27$ centímetros, compuesto de 238 páginas, numerosos grabados, dibujos, fotos en blanco y negro y figuras de línea.

Precio: España, 900 ptas., 13 \$ USA.

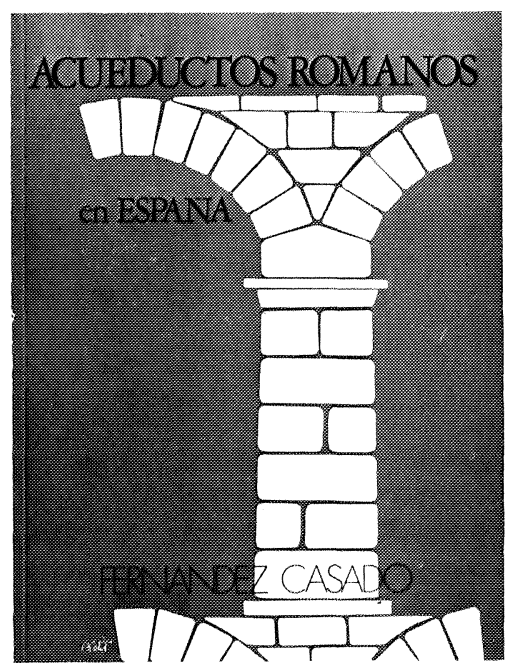

